\title{
AN EXPERIMENTAL AND NUMERICAL INVESTIGATION OF TURBULENT CATALYTICALLY STABILIZED CHANNEL FLOW COMBUSTION OF HYDROGEN/AIR MIXTURES OVER PLATINUM
}

\author{
CHRISTOPH APPEL, JOHN MANTZARAS, ROLF SCHAEREN, ROLF BOMBACH, \\ BEAT KAEPPELI AND ANDREAS INAUEN \\ Paul Scherrer Institute \\ Combustion Research \\ CH-5232 Villigen-PSI, Switzerland
}

\begin{abstract}
The turbulent catalytically stabilized combustion (CST) of fuel-lean hydrogen/air mixtures over platinum was investigated experimentally and numerically in channel-flow configurations. Experiments were performed in an optically accessible catalytic channel reactor, established by two Pt-coated ceramic plates $300 \mathrm{~mm}$ long and placed $7 \mathrm{~mm}$ apart, with incoming Reynolds numbers of 15,000 and 30,000. Planar laserinduced fluorescence of the $\mathrm{OH}$ radical was used to monitor the onset of homogeneous (gas-phase) ignition, one-dimensional (across the $7 \mathrm{~mm}$ transverse direction) Raman measurements of major species and temperature assessed the turbulent scalar transport, laser doppler velocimetry yielded the inlet velocity and turbulence, and thermocouples embedded beneath the catalyst provided the surface temperature distribution. Computations were carried out with a two-dimensional elliptic fluid mechanical code that included elementary heterogeneous and homogeneous chemical reaction schemes. Three different low-Reynolds number near-wall turbulence models were examined, in conjunction with a thermochemistry model that included a presumed-shape (Gaussian) probability density function approach for the gaseous reactions and a laminar-like closure for catalytic reactions. Comparisons between predictions and measurements have shown that key CST issues, such as catalytic fuel conversion and onset of homogeneous ignition, are strongly dependent on the particular turbulence model. Moreover, the discrepancies between predictions and measurements were ascribed to the capacity of the various turbulence models to capture the strong flow laminarization induced by the heat transfer from the hot catalytic surfaces. A turbulence model that yields good agreement with the measurements is presented as particularly suited for CST. Experiments and predictions have shown that a continuous increase of the turbulent transport led to incomplete combustion through the gaseous reaction zone with subsequent catalytic conversion of the leaked fuel and, finally, to extinction of the gaseous flame.
\end{abstract}

\section{Introduction}

The development of multidimensional CFD models is essential for the advancement of catalytically stabilized combustion (CST) to commercial systems. The numerical models should be capable of reproducing crucial CST characteristics such as light-off, catalytic fuel conversion, and-for safety reasonsthe onset of homogeneous ignition. Elementary heterogeneous reaction schemes for simple fuels $\left(\mathrm{CH}_{4}\right.$, $\mathrm{CO}$, and $\mathrm{H}_{2}$ ) over Pt were developed and tested in their capacity to reproduce measured light-off characteristics and minor species fluxes [1-3], whereas combined hetero-/homogeneous schemes for $\mathrm{CH}_{4}$ and $\mathrm{H}_{2}$ over $\mathrm{Pt}$ were validated recently $[4,5]$. In particular, the experimental and numerical investigation of Appel et al. [5] assessed the homogeneous ignition capabilities of various $\mathrm{H}_{2} / \mathrm{O}_{2}$ hetero-/homogeneous reaction schemes. Analytical studies have further elucidated the dependence of homogeneous ignition on the controlling parameters of the channel-flow configuration [6].

The flow field in all previous studies was laminar. However, in high-pressure CST systems (e.g., gas turbines), the incoming Reynolds numbers (based on the catalytic channel hydraulic diameter) range from $\sim 5,000$ to $\sim 35,000[7]$, thus requiring the development and validation of turbulent CST models. Such models should describe the transitional channel entry flow in the presence of both surface and gaseous combustion. Turbulence enhances the transport toward-or-away the catalyst and the induced thermochemical fluctuations augment the average gaseous reaction rates; the heat transfer from the hot catalytic surfaces and the exothermicity of the gaseous pathway can, in turn, result in strong laminarization of the turbulent flow $[8,9]$. For the high CST Reynolds numbers, direct numerical simulations are currently not possible, whereas large eddy simulations have subgrid model limitations in wall-bounded flows [10]. Most entry channel flow 


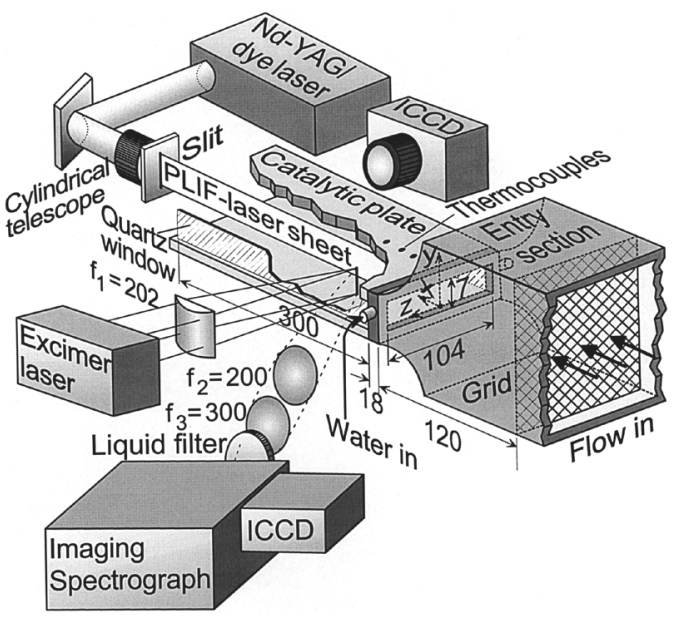

FIG. 1. Schematic of the turbulent channel-flow catalytic combustion test rig and the OH-PLIF/Raman setup. All distances are in millimeters. The combustor volume is delineated by the $300 \times 104 \times 7 \mathrm{~mm}^{3}$ enclosure.

models have, therefore, focused on low Reynolds number (LR) variants of $k-\varepsilon$ turbulence models. $\mathrm{Nu}$ merical modeling of the underlying hetero-/homogeneous processes in turbulent channel flows-with emphasis on homogeneous ignition-was reported recently in Mantzaras et al. [11].

The present study undertakes an experimental and numerical investigation of $\mathrm{H}_{2}$ /air turbulent CST over Pt, with main objectives to assess the CST applicability of various near-wall turbulence models and to understand the coupling of turbulence and hetero-/homogeneous combustion. Experiments have been performed in an optically accessible catalytic channel combustor with incoming Reynolds numbers of 15,000 and 30,000. Planar laser-induced fluorescence (PLIF) of the OH radical monitored the onset of homogeneous ignition, one-dimensional Raman measurements of major species and temperature assessed the turbulent scalar transport, laser Doppler velocimetry (LDV) yielded the inlet velocity, and thermocouples embedded beneath the catalyst provided the surface temperature. The numerical predictions included three LR turbulence models in conjunction with the thermochemistry model developed in Ref. [11].

\section{Experimental}

\section{Burner Geometry}

The turbulent CST test rig is depicted in Fig. 1. The channel reactor consisted of two horizontal $\mathrm{Si}[\mathrm{SiC}]$ ceramic plates $(300 \mathrm{~mm}$ long, placed $7 \mathrm{~mm}$ apart) and two quartz windows spring-pressed against the ceramic plates. Plasma vapor deposition was used to coat the inner $\mathrm{Si}[\mathrm{SiC}]$ surfaces with a $1.5 \mu \mathrm{m}$ thick non-porous $\mathrm{Al}_{2} \mathrm{O}_{3}$ layer, followed by a $2.2 \mu \mathrm{m}$ thick Pt layer. The surface temperature along the streamwise plane of symmetry was measured with S-type thermocouples (12 for each plate) embedded $0.9 \mathrm{~mm}$ beneath the catalytic surfaces through holes eroded from the outer plate surfaces; an additional retractable thermocouple monitored the inlet temperature $(x=0)$ of the gas. In CST of diffusionally imbalanced fuels with Lewis numbers Le $<1$ (e.g., $\mathrm{H}_{2}$ ), the catalyst can reach superadiabatic temperatures, which are more pronounced at the upstream channel locations (see Ref. [12]). Such operation compounded the experiments, since-as tests revealed - the flame anchored directly at the hot entrance. To suppress the high entry temperatures, a cooling/heating arrangement was adopted: the reactor entry was contacted to an $18 \mathrm{~mm}$ long water-cooled metal section (see Fig. 1), whereas the rear $240 \mathrm{~mm}$ of the ceramic plates was heated by two resistive heaters (to counteract the end section heat losses) positioned above the ceramic plates. The air and hydrogen flow rates were measured with two Brooks mass flow controllers. The reactants were mixed and brought to the reactor through a duct with a $y$ contraction ratio of $3: 1$. A metal grid $\left(2 \mathrm{~mm}^{2}\right.$ mesh) was positioned $120 \mathrm{~mm}$ upstream of the water-cooled section to create turbulence. The boundary layer was tripped at the upstream part of the water-cooled section.

\section{Laser Diagnostics}

The OH PLIF setup consisted of a frequencydoubled Nd:YAG pulsed laser (Quantel YG781C20), which pumped a tunable dye laser (Quantel TDL50). The resulting $285 \mathrm{~nm}$ radiation had a pulse energy of $0.5 \mathrm{~mJ}$ and was transformed into a vertical laser sheet that propagated in a counterflow direction, from the reactor's exhaust (Fig. 1). The fluorescence of both (1-1) and (0-0) transitions at 308 and $314 \mathrm{~nm}$, respectively, was collected at $90^{\circ}$ through one of the windows with an intensified CCD camera (LaVision FlameStar 2F, $576 \times 384$ pixels) equipped with a bandpass filter at $310 \mathrm{~nm}$. One-hundred images were integrated to increase the signalto-noise ratio. The PLIF was calibrated with absorption measurements performed with the laser sheet crossing laterally (z-direction) through both quartz windows, as in previous $\mathrm{CH}_{4}$ /air studies [4].

In the single-shot Raman measurements, a twostage narrowband $\mathrm{KrF}$ excimer laser (Lambda Physik Compex $150 \mathrm{~T}$ ) was tuned to $248 \mathrm{~nm}$ with a pulse energy of $230 \mathrm{~mJ}$. The beam was focused to a $0.3 \mathrm{~mm}$ thick vertical line inside the combustor with a cylindrical lens (Fig. 1). The focal line spanned the $7 \mathrm{~mm}$ transverse extent of the channel and was offset 


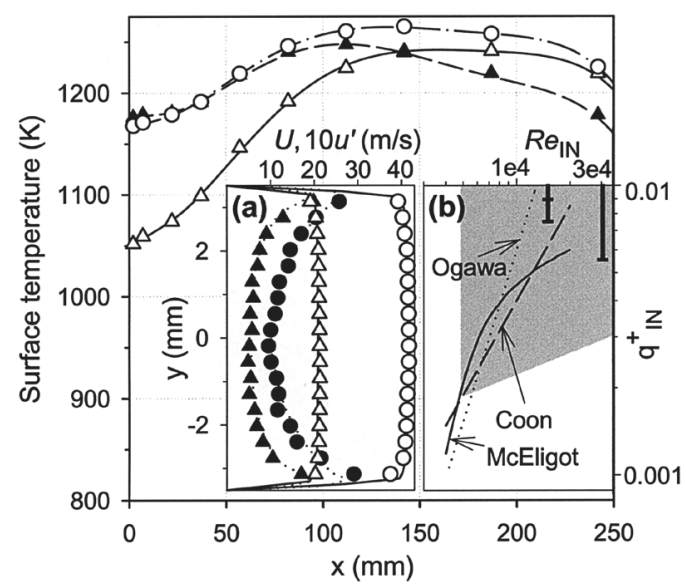

FIG. 2. Main figure, measured surface temperature profiles: case 1, open triangles; case 2, solid triangles; case 3, open circles. (Inset a) Measured inlet mean velocities (open symbols) and inlet streamwise turbulence intensities $u$ (solid symbols): cases 1 and 2, triangles; case 3, circles. Cases 1 and 2 have common mean velocity and turbulence levels. (Inset b) Regimes of laminarization as a function of inlet Reynolds number $R e_{\mathrm{IN}}$ and non-dimensional heat transfer parameter $q_{\mathrm{IN}}^{+}=q_{\mathrm{W}} /\left(\rho U c_{\mathrm{p}} T\right)_{\mathrm{IN}}$, with $q_{\mathrm{W}}$ the wall heat flux and $c_{\mathrm{p}}$ the specific heat of the mixture at constant pressure. The lines (Refs. [16-18]) delineate laminarization: in the area above the lines the flow laminarizes and in the area below the turbulence increases. The shaded area presents an estimate of the regimes applicable in CST.

laterally $(z=15 \mathrm{~mm})$ to increase the collection angle and minimize beam steering. As the combustion in the channel was strongly two-dimensional (except $\sim 15 \mathrm{~mm}$ from the windows), the offset had no implications to the comparisons with two-dimensional numerical predictions. The Raman signal was collected at $50^{\circ}$ and focused into a $25 \mathrm{~cm}$ imaging spectrograph (Chromex 250i) equipped with a second intensified CCD camera, identical to that of the PLIF setup. The spectral dispersion ranged from 1250 to $5000 \mathrm{~cm}^{-1}$, allowing observation of all major species $\left(\mathrm{N}_{2}, \mathrm{O}_{2}, \mathrm{H}_{2} \mathrm{O}\right.$, and $\left.\mathrm{H}_{2}\right)$; fluorescence interferences from $\mathrm{O}_{2}$ hot-band transitions precluded quantitative $\mathrm{O}_{2}$ measurements. Measurements were acquired at $15 \mathrm{~mm}$ intervals by traversing axially the test rig. Harmonic oscillator Raman cross-section variations were used for the diatomic species [13] and the data from Eisenberg for $\mathrm{H}_{2} \mathrm{O}$ [14]. The temperature was assessed from the $\mathrm{N}_{2}$ Raman data. Raman measurements at distances less than $0.6 \mathrm{~mm}$ from both walls were discarded due to low signalto-noise ratio. The Raman setup was similar to that of earlier laminar $\mathrm{H}_{2}$ /air CST studies [5].

Finally, the LDV setup consisted of a Dantec 57N20BSA system with a Coherent Innova $300 \mathrm{Ar}$ Ion laser and a photomultiplier tube in backscatter mode. The flow was seeded with paraffin oil and the inlet axial velocity profile $(x=0, z=0)$ was measured.

\section{Numerical}

\section{Thermochemistry Modeling}

A two-dimensional elliptic code based on a finitevolume approach was used. Favre-averaged modeled transport equations were solved for the continuity, momentum, and all first- and second-order scalar moments (mean values, variances, and covariances of the species mass fractions and the total enthalpy; see Ref. [11]). The transport equation for every gaseous variable $\varphi$ was

$$
\begin{gathered}
\frac{\partial(\bar{\rho} \tilde{u} \tilde{\varphi})}{\partial x}+\frac{\partial(\bar{\rho} \tilde{v} \tilde{\varphi})}{\partial y}-\frac{\partial}{\partial x}\left(\Gamma_{\text {eff }} \frac{\partial \tilde{\varphi}}{\partial x}\right) \\
-\frac{\partial}{\partial y}\left(\Gamma_{\text {eff }} \frac{\partial \tilde{\varphi}}{\partial y}\right)=\tilde{S}_{\varphi}
\end{gathered}
$$

with

$$
\Gamma_{\text {eff }}=\Gamma+\mu_{\mathrm{t}} / \sigma_{\varphi} \text { and } \mu_{\mathrm{t}}=\bar{\rho} c_{\mu} f_{\mu} \tilde{k}^{2} / \tilde{\varepsilon}
$$

To evaluate the average species chemical reaction rates $\left(\tilde{S}_{i}\right.$, where $i$ is the gaseous species), a presumed Gaussian joint probability density function (pdf) was used for every elementary reaction. This approach (applied successfully in $\mathrm{H}_{2}$ /air jet flames [15]) retained the elementary level chemistry description, which - in the absence of validated reduced hetero-/ homogeneous schemes-was necessary to account for the important hetero-/homogeneous coupling of minor species (radicals). For a bimolecular reaction, the joint pdf depended on nine moments established by the temperature and the two species mass fractions; the pdf was truncated to maintain realizable values for the species mass fractions and temperature. Lookup tables of mean reaction rates were precalculated and used during the numerical solution.

In heterogeneous combustion, Favre averaging of the surface chemical source terms did not introduce difficulties; the large thermal inertia of the ceramic plates removed the surface temperature fluctuations and hence the major reaction nonlinearity (exponential Arrhenius term). The mean heterogeneous reaction rates were then treated with a laminar-like closure [11], evaluated at their corresponding mean values, $\tilde{s}_{k}=s_{k}\left(T_{\mathrm{W}}, \tilde{Y}_{k}, \tilde{\Theta}_{m}\right)$.

\section{Turbulence Modeling}

Turbulence closure was achieved via LR $\tilde{k}-\tilde{\varepsilon}$ models. The ability of various turbulence models to capture key CST issues, such as catalytic conversion and onset of homogeneous ignition, has not been reported in the literature. Fig. $2 \mathrm{~b}$ (adapted from 
non-reacting heated channel flows) delineates laminarization as a competition between the incoming Reynolds number $\left(R e_{\mathrm{IN}}\right)$ and a non-dimensional heat transfer parameter $\left(q_{\mathrm{IN}}^{+}\right)$: the lines [16-18] demarcate an upper laminarizing domain due to strong heating and a lower non-laminarizing domain; the shaded area is our assessment of the CST-relevant regime.

The key features of the employed LR models are given next. The relevant source terms in the $\tilde{k}$ and $\tilde{\varepsilon}$ equations are

$$
\begin{aligned}
& \tilde{S}_{k}=P_{k}+G_{k}-\bar{\rho} \tilde{\varepsilon} \\
& \quad \text { and } \tilde{S}_{\varepsilon}=\left(C_{\varepsilon 1} P_{k}+C_{\varepsilon 2} G_{k}-f_{3} C_{\varepsilon 3} \bar{\rho} \tilde{\varepsilon}\right) \tilde{\varepsilon} / \tilde{k}
\end{aligned}
$$

respectively. The first approach was the two-layer model of Chen and Patel [19], developed with prime goal to provide a computationally efficient tool by splitting the flow domain into two zones. In the outer zone, both $\tilde{k}$ and $\tilde{\varepsilon}$ equations were solved and in the inner zone (which included the viscous sublayer, the buffer layer, and part of the fully turbulent layer), only the $\tilde{k}$ equation was solved and $\tilde{\varepsilon}$ and $\mu_{\mathrm{t}}$ were calculated from $\tilde{\varepsilon}=\tilde{k}^{3 / 2} / \ell_{\varepsilon}$ and $\mu_{\mathrm{t}}=\bar{\rho} C_{\mu} \tilde{k}^{1 / 2} \ell_{\mu} ; \ell_{\mu}$ and $\ell_{\varepsilon}$ were length scales containing near-wall damping in terms of a local turbulent Reynolds number $R_{k}=\tilde{k}^{1 / 2} y / v$. The two layers were matched at $R_{k} \approx$ 250 .

The other two approaches were full LR models, involving integration of both $\tilde{k}$ and $\tilde{\varepsilon}$ equations directly to the wall. The first model (further denoted as Lin [20]) was developed and tested in channel flows with and without wall heating. Special attention was given to properly reproduce the near-wall asymptotic behavior. The second full LR model (further denoted as Ezato [8]) is a recent improvement of existing LR thermal entry-flow models that has been validated in strongly laminarizing turbulent channel flows. In all models, the turbulent species Schmidt numbers were $0.9, C_{\mu}=0.09$, and $C_{\varepsilon 2}=$ 1.0. The damping functions $f_{3}$ and $f_{\mu}$ that determined the degree of laminarization and the constants $C_{\varepsilon 1}$ and $C_{\varepsilon 3}$ are given in the original model publications.

\section{Numerical Approach}

Computations were performed up to $x=$ $250 \mathrm{~mm}$, since homogeneous ignition occurred well within this streamwise extent. In the two-layer model, an orthogonal staggered grid of $300 \times 60$ points (in $x$ and $y$ ) was used over half the domain $\left(250 \times 3.5 \mathrm{~mm}^{2}\right)$; gravity was not included as it was not important for the Reynolds numbers of this study. In the full LR models, a $y$-grid of 140 points was sufficient to produce a grid-independent solution (the near-wall node was positioned at $y^{+}=0.5$, as opposed to $y^{+}=15$ in the two-layer model). The inlet conditions were uniform properties for the species mass fractions and temperature, and variable (measured) profiles for the mean axial velocity and $\tilde{k}$ (the inlet $\tilde{\varepsilon}$ was deduced from the correlation of Ref. [21]). At the wall $(y=0)$, the measured temperature profile (average between both plate distributions) was used as energy boundary condition; in both full LR models $\tilde{\varepsilon}=2 v(\partial \sqrt{k} / \partial y)^{2}$ at $y=0[8,20]$. Finally, zero-Neumann boundary conditions [11] were applied at the plane of symmetry $(y=3.5 \mathrm{~mm})$ and the outlet $(x=250 \mathrm{~mm})$.

\section{Chemical Kinetics}

For surface chemistry, the $\mathrm{H}_{2} / \mathrm{O}_{2}$ scheme over $\mathrm{Pt}$ by Deutschmann [1] was employed (five surface species, six gaseous species, and 16 reactions). The surface site density was $2.7 \times 10^{-9} \mathrm{~mol} / \mathrm{cm}^{2}$, simulating polycrystalline platinum [1,5]; the thick $(2.2 \mu \mathrm{m})$ platinum layer on top of a non-porous $\mathrm{Al}_{2} \mathrm{O}_{3}$ layer, closely resembled such a surface. For gaseous combustion, the $\mathrm{H}_{2} / \mathrm{O}_{2}$ scheme of Warnatz et al. [22] (38 reactions and nine species including $\mathrm{N}_{2}$ ) was used. The above schemes have been validated in their capacity to reproduce measured catalytic fuel conversion and homogeneous ignition in laminar channel CST [5]. Gaseous and surface reaction rates were evaluated with CHEMKIN [23] and SurfaceCHEMKIN [24], respectively. The CHEMKIN database was also used for thermodynamic and laminar transport data.

\section{Results and Discussion}

Experiments were performed at atmospheric pressure for three cases with the following equivalence ratios $(\varphi)$, mean inlet velocities $\left(U_{\mathrm{IN}}\right)$, and inlet Reynolds numbers. Case 1 was $\varphi=0.18, U_{\text {IN }}=$ $20 \mathrm{~m} / \mathrm{s}$, and $R e_{\mathrm{IN}}=15,390$; case 2 was $\varphi=0.24$, $U_{\mathrm{IN}}=20 \mathrm{~m} / \mathrm{s}$, and $R e_{\mathrm{IN}}=15,080$; and case 3 was $\varphi=0.24, U_{\mathrm{IN}}=40 \mathrm{~m} / \mathrm{s}$, and $R e_{\mathrm{IN}}=30,160$. The measured profiles of $U_{\text {IN }}$ and turbulence intensity $u^{\prime}$ are illustrated in Fig. 2a; the measured surface temperature is in Fig. 2. The measured inlet temperature was in all cases $T_{\mathrm{IN}}=300 \mathrm{~K}$. The solid bars in Fig. $2 \mathrm{~b}$ position the experiments on the $R e_{\mathrm{IN}}-$ $q_{\mathrm{IN}}^{+}$parametric plot; as seen, they span the domain of laminarization. The leaner case 1 had the lowest possible $\varphi$ that yielded (experimentally) ignition and was chosen to test the models' capability at extreme conditions. An additional reason was to examine gaseous combustion effects on flow laminarization; Cheng et al. [9] reported strong flow laminarization with increasing $\varphi$ in near-wall gaseous $\mathrm{H}_{2}$ combustion. Fig. 3 presents PLIF-measured and numerically predicted mean $\mathrm{OH}$ distributions. The stability and reproducibility of the measured flame shapes and anchoring positions was excellent over extended measuring times. The predictions of Fig. 3 refer only to those models that were able to yield homogeneous ignition; the onset of homogeneous ignition (yellow 


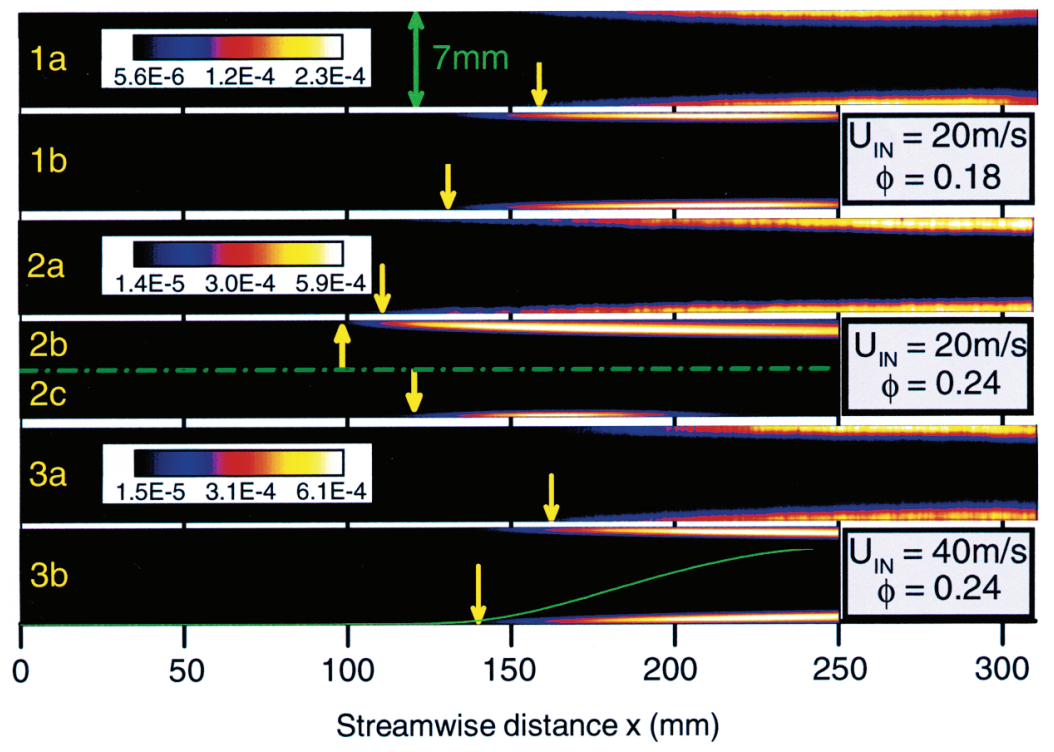

FIG. 3. Measured (1a, 2a, and 3a) and predicted (1b, 2b, 2c, and 3b) distributions of the mean OH mole fraction for cases 1,2 , and 3 , respectively. The onset of homogeneous ignition (shown by the yellow arrows) in both measurements and predictions is defined by the sharp rise of the $\mathrm{OH}$ profile, as indicated in Fig. 3b. The green curve of Fig. 3b provides the integrated (over the transverse extent) $\mathrm{OH}$ levels; the intersection of the tangent to the inflection point of this curve with the wall determines homogeneous ignition. The numerical predictions refer to the following models: $1 b, 2 b$, and 3b, Ezato; 2c, Lin. In $2 \mathrm{~b}$ and 2c, only half of the channel is shown. The color bars define the $\mathrm{OH}$ mole fraction levels.

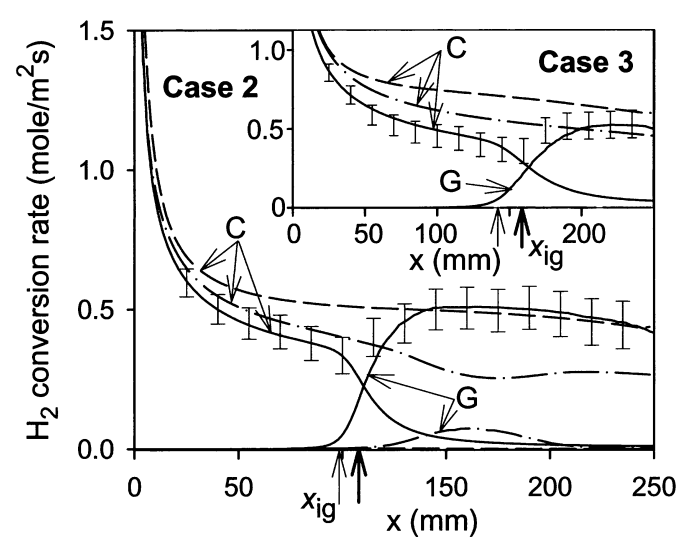

FIG. 4. Predicted catalytic $(C)$ and gaseous $(G)$ hydrogen local conversion rates for cases 2 and 3 (inset): dashed lines, two-layer model; dashed-dotted lines, Lin model; solid lines, Ezato model. The gaseous conversion of the twolayer model is nearly zero in case 2 , and the gaseous conversion of both the two-layer and Lin models is nearly zero in case 3 . The measured combined (catalytic plus gaseous) hydrogen conversions are presented with vertical bars. The vertical arrows indicate the location of homogeneous ignition $\left(x_{\mathrm{ig}}\right)$ according to Fig. 2. Thin arrows, predictions from Ezato's model; thick arrows, measurements. arrows) is defined by the sharp rise of the $\mathrm{OH}$ profile (see green line in Fig. 3 [3b]). Fig. 3 indicates that the LR model of Ezato captured (within 16\%) the homogeneous ignition distance $\left(x_{\mathrm{ig}}\right)$ and the postignition flame shape in all cases, the model of Lin predicted ignition only for case 2 (Fig. 3 [2c]), however, with a resulting very short flame length and, finally, the two-layer model yielded no homogeneous ignition in all cases.

To understand the origin of the model discrepancies, an analysis of the turbulent transport processes was carried out. Fig. 4 presents the computed local $\mathrm{H}_{2}$ catalytic fuel conversion $(C)$ and the integrated (over the transverse direction) $\mathrm{H}_{2}$ gaseous conversion $(G)$ for cases 2 and 3 along with the measured combined (catalytic plus gaseous) conversion. In case 2 , the two-layer model clearly overpredicted the catalytic fuel conversion over the preignition distance, the model of Lin overpredicted to a smaller degree the preignition catalytic fuel conversion and failed to capture the total fuel conversion in the postignition zone and, finally, the model of Ezato captured the fuel conversion over the entire channel length, notwithstanding the small differences between measured and predicted $x_{\mathrm{ig}}$. The discrepancies of the two-layer and Lin models were even more pronounced in the higher $R e_{\mathrm{IN}}$ case 3 (see inset of Fig. 4), whereas case 1 (not shown) had a behavior similar to that of case 3 , albeit with lower absolute 


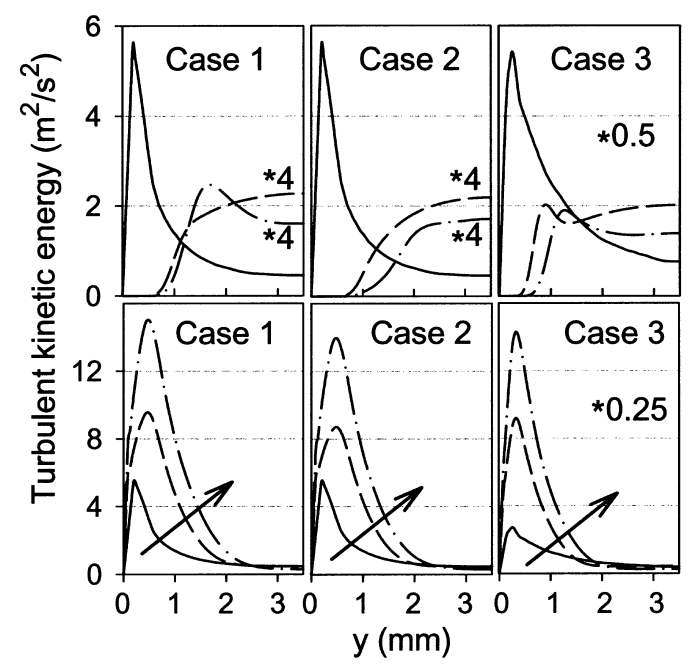

FIG. 5. Transverse profiles of turbulent kinetic energy at three selected streamwise distances for the models of Ezato (top row) and Lin (bottom row); $x=0$ (solid lines), $x=100$ (dashed lines), and $x=200 \mathrm{~mm}$ (dashed-dotted lines). The wall is located at $y=0$. For reasons of clarity, the turbulent kinetic energy in case 3 has been multiplied by a factor of 0.5 (Ezato) and 0.25 (Lin); the profiles at $x=100$ and $200 \mathrm{~mm}$ of cases 1 and 2 in Ezato's model have been multiplied by a factor of 4 . The arrows point to the direction of increasing $x$.

$\mathrm{H}_{2}$ conversion. The above model performance is elaborated in Fig. 5, presenting computed transverse profiles of the turbulent kinetic energy at three selected streamwise distances for the models of Lin and Ezato. The corresponding profiles of the twolayer model are similar to those of the Lin model, but with up to $80 \%$ higher values at the downstream axial positions. Concentrating on cases 2 and 3, the turbulent kinetic energy decreased (laminarized) along the streamwise direction in the Ezato model whereas it increased in the Lin and two-layer models. The enhanced $\tilde{k}$ of the last two models resulted in an increased $\mathrm{H}_{2}$ transport toward the wall (see the effective transport coefficient $\Gamma_{\text {eff }}$ of equation 2); the surface chemistry was fast enough to cope with the increased transport, yielding the increased preignition catalytic $\mathrm{H}_{2}$ conversion shown in Fig. 4. The lack of flow laminarization had a direct impact on homogeneous ignition. First, the enhanced transport resulted in increased catalytic $\mathrm{H}_{2}$ conversion for the two-layer and Lin models (reducing the fuel availability for the gaseous pathway) and in more efficient removal of heat from the near-wall ignitable mixture: both effects inhibited homogeneous ignition as also manifested by the later predicted ignition of case 2 in Lin's model. Second, the increased transport rates resulted in reduced residence times through the potential near-wall gaseous combustion zone. The latter effect was first addressed in the stagnation point flow catalytic studies of Law and Sivashinsky [25]: for fuels with $L e<1$, an increase of the strain rate pushed the flame against the wall, led to incomplete gaseous fuel combustion followed by catalytic conversion of the leaked fuel, and eventually led to extinction. In our recent turbulent CST numerical studies [11], increased fuel leakage through the gaseous zone was obtained with increased turbulence intensity. The increased transport resulted in lack of homogeneous ignition for both two-layer and Lin models, except in case 2 where a very short flame was established in the model of Lin. Case 2 had the strongest laminarization due to the lowest $R e_{\text {IN }}$ (compared to case 3) and due to the higher wall heat transfer (compared to case 1, see the wall temperature profiles of Fig. 2); in addition, since $\varphi$ was higher, the establishment of a potentially stronger flame would further laminarize the flow [9]. These two factors of case 2 allowed for an initial flame development in Lin's model; however, as there was no laminarization and $\tilde{k}$ increased farther downstream, the flame extinguished shortly. On the other hand, the continuous laminarization of Ezato's model allowed for higher flame residence times, a sustained near-wall gaseous combustion, and a correct prediction of the flame length; furthermore, the $k$ levels were practically zero in the near-wall flame zone. The present results have also shown that the two-layer predictions of our previous work [11] reproduced only qualitatively the underlying hetero-/ homogeneous processes; the quantitative description required a validated model such as that of Ezato.

Figure 6 presents Raman-measured and predicted (Ezato model) transverse profiles of the mean temperature, $\mathrm{H}_{2}$ and $\mathrm{H}_{2} \mathrm{O}$ mole fractions at three selected streamwise distances. The very low $\mathrm{H}_{2}$ wall levels indicate that the catalytic fuel conversion was practically mass transport limited in all cases. The overall good agreement between measurements and predictions provided a direct validation for the turbulent transport of Ezato's model. In addition, the symmetry of the channel-flow combustion was evident. As seen from Fig. 6, the species and thermal boundary layers were narrower in the higher $R e_{\text {IN }}$ case 3 and the fuel depletion was stronger in the lower $R e_{\text {IN }}$ cases 1 and 2 . The degree of turbulence determined the type of fuel conversion (heterogeneous or homogeneous). In the higher turbulence intensity cases 1 and 3, fuel leakage through the gaseous combustion zone was supported from both predictions and measurements: the transverse wall gradient of $\mathrm{H}_{2}$ was non-zero in cases 1 and 3 (Fig. 6 $[1 \mathrm{c}, 3 \mathrm{c}]$ ), whereas in the strongly laminarizing case 2 the transverse wall gradient was practically zero over the postignition zone (Fig. 6 [2c]), indicating no fuel leakage through the gaseous zone. Therefore, both cases 1 and 3 had a combined heterogeneous and homogeneous fuel conversion in the 


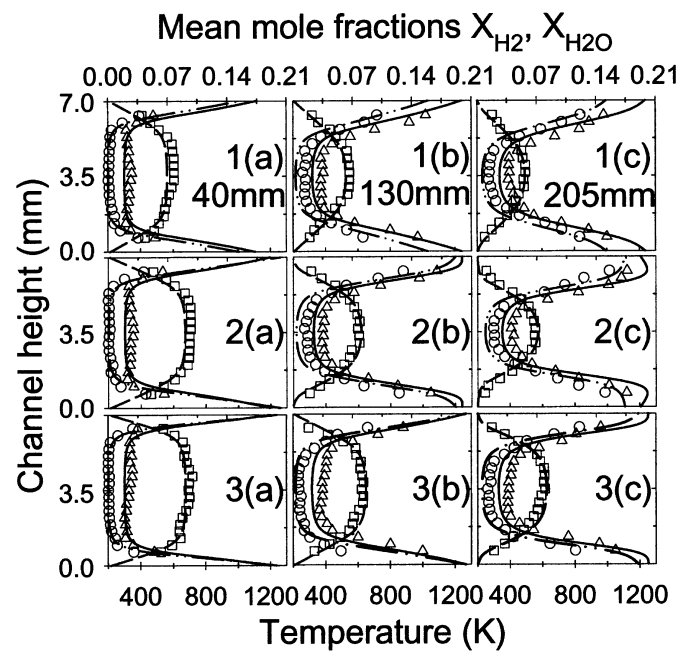

FIG. 6. Transverse profiles of measured and predicted (Ezato model) mean values of temperature, $\mathrm{H}_{2}$ and $\mathrm{H}_{2} \mathrm{O}$ mole fractions, at three selected streamwise distances: (a) $x=40 \mathrm{~mm}$, (b) $x=130 \mathrm{~mm}$, and (c) $x=205 \mathrm{~mm}$, for cases 1 (top), 2 (middle), and 3 (bottom). Each measurement is the average over 600 laser shots. Measurements: $\mathrm{H}_{2}$ (squares), $\mathrm{H}_{2} \mathrm{O}$ (circles), and temperature (triangles). Predictions: $\mathrm{H}_{2}$ (dashed lines), $\mathrm{H}_{2} \mathrm{O}$ (dashed double-dotted lines), and temperature (solid lines).

postignition zone (see also inset of Fig. 4). Due to the stronger gaseous combustion of case 2 , the predicted near-wall gas temperature at the downstream distances exceeded the wall temperature (Fig. 6 $[2 \mathrm{c}])$. The small underpredictions of $\mathrm{H}_{2} \mathrm{O}$ and temperature in the channel core and the corresponding overpredictions of $\mathrm{H}_{2}$ (in conjunction with the somewhat earlier homogeneous ignition predictions) suggested that the model of Ezato overpredicted mildly the degree of laminarization. The predictions of both the Lin and two-layer models resulted in narrower scalar boundary layers and steeper transverse scalar wall gradients than those measured in Fig. 6.

Transverse profiles of the normalized $\mathrm{H}_{2}$ root mean square (rms) fluctuations are shown in Fig. 7 at two selected streamwise positions. The measurements indicated that, for a given case, the profile peaks moved away from the wall with increasing $x$. Alternately, for a given $x$, the higher $R e_{\mathrm{IN}}$ case 3 had its rms peak closer to the wall. These trends were captured only by the Ezato model, which also reproduced the measured profile shapes. Even though the rms shapes were well predicted by the Ezato model, the absolute rms values were underpredicted. This could be attributed to experimental factors due to increased signal-to-noise ratio of the single-shot Raman data and to inherent limitations of the $k-\varepsilon$ turbulence models, which typically underpredict scalar fluctuations [26]. Probability density

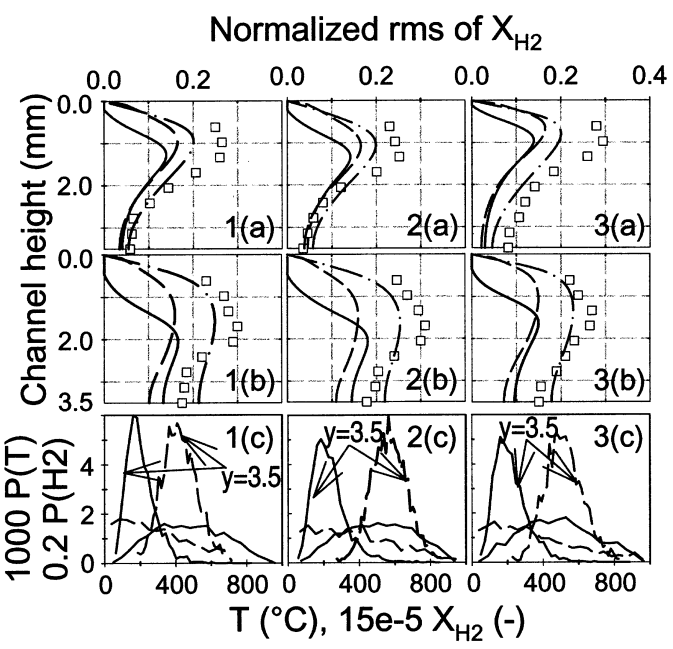

FIG. 7. Transverse profiles of the normalized root mean square $\mathrm{H}_{2}$ fluctuations: (a) $x=40$ (top row) and (b) $x=$ $130 \mathrm{~mm}$ (middle row), for cases 1,2 , and 3 . The normalization is carried out with the corresponding mean $\mathrm{H}_{2}$ mole fraction at the plane of symmetry $(y=3.5 \mathrm{~mm})$. Due to symmetry, only half the channel is shown. Square symbols, measurements; lines, predictions Ezato (solid lines), Lin (dashed lines), and two-layer (dashed-dotted lines). Bottom row (c), probability density functions of the $\mathrm{H}_{2}$ mole fraction, $P\left(\mathrm{H}_{2}\right)$, and temperature, $P(T)$, at $x=130 \mathrm{~mm}$ and two selected transverse positions $y=0.8$ and $3.5 \mathrm{~mm}$, for cases 1, 2, and 3. Solid lines, temperature; dashed lines, hydrogen.

functions of the temperature and hydrogen mole fraction are provided in Fig. 7 (bottom line). The scalar pdf shapes maintained a generally symmetric character, consistent with the presumed Gaussian pdf closure of the thermochemistry model.

\section{Conclusions}

The experimental and numerical investigation of the turbulent CST of $\mathrm{H}_{2}$ /air mixtures in Pt-coated channels has exemplified the importance of nearwall turbulence models that capture the strong-flow laminarization induced by the heat transfer from the catalytic walls. The laminarization and, therefore, the magnitude of turbulent transport were crucial for the assessment of both catalytic fuel conversion and homogeneous ignition. Predictions with three different low Reynolds number turbulence models-adapted from the literature without any modifications-have shown the strong sensitivity of the hetero-/homogeneous processes on the particular turbulence model. A strong flow laminarization reduced the catalytic fuel conversion and aided the onset of homogeneous ignition. An increase in the turbulent transport rates resulted in incomplete fuel combustion through the gaseous zone, subsequent 
catalytic conversion of the leaked fuel, and finally, to flame extinction. The experiments have shown that the model of Ezato reproduced with good accuracy both the preignition catalytic conversion and the onset of homogeneous ignition, although it overpredicted mildly the degree of laminarization.

\section{Nomenclature}

$f_{\mu}, f_{3} \quad$ damping functions (equations 2 and 3 )

$k \quad$ turbulent kinetic energy

$P_{k}, G_{k} \quad$ production terms of turbulent kinetic energy (equation 3)

$\tilde{S}_{i}, \tilde{s}_{k} \quad$ gas-phase and surface average chemical source terms

$u, v \quad$ streamwise and transverse velocity components

$u^{\prime} \quad$ streamwise turbulence intensity

$y^{+} \quad$ non-dimensional distance $\left(y^{+}=y u^{+} / v\right.$; $u^{+}$is the wall friction velocity)

$T_{\mathrm{W}} \quad$ wall temperature

$\Gamma_{\text {eff, }} \Gamma \quad$ effective transport coefficient, laminar transport coefficient (equation 2)

$\tilde{\varepsilon} \quad$ dissipation rate of the turbulent kinetic energy

$\Theta_{k} \quad$ surface coverage of species $k$

$\mu_{\mathrm{t}}, v \quad$ turbulent viscosity (equation 2), laminar kinematic viscosity

$\rho, \sigma_{\varphi} \quad$ density, turbulent Prandtl/Schmidt number

Superscripts

$\sim,-\quad$ Favre and Reynolds averaging

$$
\text { Acknowledgments }
$$

Support was provided by the Swiss Federal Office of Energy (BFE), Office of Education and Technology (BBT), and Alstom Power Technology of Switzerland. We are indebted to Dr. Paul Papas of EPFL for the LDV measurements.

\section{REFERENCES}

1. Deutschmann, O., Schmidt, R., Behrendt, F., and Warnatz, J., Proc. Combust. Inst. 26:1747 (1996).

2. Hickman, D. A., and Schmidt, L. D., AIChE 39(7):1164 (1993).
3. Bui, P. A., and Vlachos, P. R., Proc. Combust. Inst. 26:1763 (1996).

4. Dogwiler, U., Mantzaras, J., Benz, P., Kaeppeli, B., Bombach, R., and Arnold, A., Proc. Combust. Inst. 27:2275 (1998).

5. Appel, C., Mantzaras, J., Schaeren, R., Bombach, R., Inauen, A., Kaeppeli, B., Hemmerling, B., and Stampanoni, A., Combust. Flame 128:340 (2002).

6. Mantzaras, J., and Benz, P., Combust. Flame 119:455 (1999).

7. Dalla Betta, R. A., and Rostrup-Nielsen, T., Catal. Today 47:369 (1999).

8. Ezato, K., Shehata, A. M., Kunugi, T., and McEligot, D. M., ASME 121:546 (1999).

9. Cheng, R. K., Bill, R. G., and Robben, F., Proc. Combust. Inst. 18:1021 (1980).

10. Haertel, C., and Kleiser, L., J. Fluid Mech. 356:327 (1998).

11. Mantzaras, J., Appel, C., Benz, P., and Dogwiler, U., Catal. Today 59:3 (2000).

12. Pfefferle, W. C., and Pfefferle, L. D., Prog. Energy Combust. Sci. 12:25 (1985).

13. Brockhinke, A., Andresen, P., and Kohse-Höinghaus, K., Appl. Phys. B 61:533 (1995).

14. Eisenberg, S., "Raman Spektroskopie an einer Zerstäuberflamme," Diploma thesis, Max-Planck-Institut, Göttingen, 1995.

15. Bockhorn, H., Proc. Combust. Inst. 22:655 (1988).

16. Ogawa, M., Kawamura, H., Takizuka, T., and Akino, N., J. Atomic Energy Soc. Jpn 24:60 (1981).

17. Coon, C. W., and Perkins, H. C., ASME Ser. C 92:506 (1970).

18. McEligot, D. M., Coon, C. W., and Perkins, H. C., Int. J. Heat Mass Transfer 13:431 (1970).

19. Chen, H. C., and Patel, V. C., AIAA J. 26(6):641 (1988).

20. Hwang, C. B., and Lin, C. A., AIAA J. 36(1):38 (1998).

21. Wolfshtein, M., Int. J. Heat Mass Transfer 12:301 (1969).

22. Warnatz, J., Dibble, R. W., and Maas, U., Combustion, Physical and Chemical Fundamentals, Modeling and Simulation, Springer-Verlag, New York, 1996, p. 69.

23. Kee, R. J., Rupley, F. M., and Miller, J. A., Sandia report SAND89-8009B, July 1996.

24. Coltrin, M. E., Kee, R. J., and Rupley, F. M., Sandia report SAND90-8003C, July 1996.

25. Law, C. K., and Sivashinsky, G. I., Combust. Sci. Technol. 29:277 (1982).

26. Chen, C., Riley, J. J., and McMurtry, P. A., Combust. Flame 87:257 (1991). 